\title{
Hemodynamic Performance Validation of Customized Handmade Trileaflet-valved Conduits Using Taguchi Method and Likelihood Degree Estimation
}

\author{
Chia-Hung Lin, ${ }^{1,2}$ Xuan-Hao Zhang, ${ }^{1}$ Neng-Sheng Pai, ${ }^{1 *}$ \\ Jian-Xing $\mathrm{Wu},{ }^{1}$ and Chung-Dann $\mathrm{Kan}^{3}$ \\ ${ }^{1}$ Department of Electrical Engineering and Artificial Intelligence Application Research Center, \\ National Chin-Yi University of Technology, Taichung City 41170, Taiwan \\ ${ }^{2}$ Artificial Intelligence Application Research Center, National Chin-Yi University of Technology, \\ Taichung City 41170, Taiwan \\ ${ }^{3}$ Division of Cardiovascular Surgery, Department of Surgery, National Cheng Kung University Hospital, \\ College of Medicine, National Cheng Kung University, Tainan City 70101, Taiwan
}

(Received June 10, 2020; accepted November 4, 2020)

Keywords: pulmonary valve replacement, regurgitation fraction, heart pump efficiency, Taguchi method, signal-to-noise ratio, likelihood degree estimation

Transcatheter pulmonary valve replacement (TPVR) is a technique for treating valvular heart diseases or dysfunctions without the need for open-heart surgery. Commercial valve stents, such as Epic ${ }^{\mathrm{TM}}$ valved stents or mechanical heart valves, can be used to solve the problems of narrowed or leaky pulmonary valves and also to improve regurgitation flow and heart pump efficiency (HPE). However, these prosthetic valves have limitations in terms of availability, durability, and stent size for children and for meeting the needs of special subjects. To be deemed fit for new clinical cases, handmade trileaflet-valved conduits (HTVCs) provide a promising strategy for designing stents using optimal design methods for customized specifications for child and adult patients. Before clinical applications, the functions of HTVCs need to verify hemodynamic statuses, such as the regurgitation fraction $(R F), H P E$, and pressure drop, under different heart rates $(H R)$, blood flow volumes, and hypertension conditions. To reduce the number of experimental tests, the Taguchi method with signal-tonoise $(S / N)$ ratios is used to validate the assigned optimal parameter designs, which can obtain good hemodynamic performance with the desired target goals of (1) $R F \leq 20 \%$ (minimizing the objective function) and (2) $H P E \geq 80 \%$ (maximizing the objective function). In contrast to commercial valve stents, such as Epic ${ }^{\mathrm{TM}}$ valved stent and mechanical heart valve, likelihood degree estimation is also employed to quantify the degree of valve performance and verify the quality of HTVCs.

\section{Introduction}

Congenital heart diseases result in chronic pulmonary insufficiency, right ventricular dilation, and ventricular arrhythmia. Chronic valvular insufficiency is also a pathological abnormality that gradually affects the right ventricle, pulmonary artery, or pulmonary *Corresponding author: e-mail: pai@ncut.edu.tw https://doi.org/10.18494/SAM.2021.3016 
parenchyma. The reconstruction of the right ventricular outlet is a strategy to improve blood flow regurgitation and enhance the efficiency of pumping blood in sufficient volumes to the right and left lungs. ${ }^{(1,2)}$ However, postoperative pulmonary valve degeneration and calcification on previous substitutes result in pulmonary valve dysfunction with aging in both children and adults. These phenomena on previous substitutes also result in pulmonary valve dysfunction, overworked blood-pumping ventricles, and increased pulmonary vascular resistance. ${ }^{(3,4)}$ Patients also suffer from subsequent dysfunctions of both the right and left ventricles, pressure, and volume overload for the right ventricle, such as high right ventricular pressure (hypertension condition, $>60 \mathrm{mmHg})^{(5,6)}$ For different ages of patients, pulmonary valve replacement is a good strategy to treat the pulmonary valve insufficiency between the right ventricle and the pulmonary artery. Hence, previous studies have used handmade trileaflet-valved conduits (HTVCs) with expanded polytetrafluoroethylene (ePTFE) to reconstruct the right ventricular outflow tract (RVOT) without the need for open-heart surgery. ${ }^{(7-9)}$ In relation to this reason, the purpose of this study is to validate the performance of customized HTVCs for pulmonary valve reconstruction using the Taguchi method by taking into account signal-to-noise $(S / N)$ ratios under different heart rates $(H R)$, blood flow volumes, and hypertension conditions.

In clinical applications, homograft conduits are the most used substitutes for pulmonary valve replacement. Bovine jugular vein conduits and porcine valves are also employed for pulmonary valve replacement. However, these organismic substitutes do not guarantee durability as calcification occurs with age. In addition, not all homograft conduit sizes are always available for customized applications. Commercial valve stents, such as Epic ${ }^{\mathrm{TM}}$ valves and mechanical heart valves, ${ }^{(10-14)}$ can also potentially be used to improve blood flow regurgitation and hemodynamic conditions. However, these prosthetic valved conduits also have issues of availability, durability, and size limitations, thereby restricting their usage in both child and adult patients. To overcome the restrictions, previous studies ${ }^{(7-9,15)}$ have presented HTVCs that provide a large range of customized diameters for RVOT reconstructions. ePTFE has good biocompatibility (and has been approved by the Taiwan Food and Drug Administration) and possesses low tissue affinity against valve degeneration and calcification. ${ }^{(16-18)}$ In addition, HTVCs can be rapidly designed using computed tomography (CT) angiography measurement ${ }^{(7)}$ and machine-learning-based intelligent estimators, such as the metalearning-based and cascade correlation learning models proposed in previous studies. ${ }^{(16,19)}$ Trileaflet sizes and HTVC diameters can be determined by CT imaging. Machine-learning-based intelligent estimators are employed to determine optimal pulmonary leaflet parameters, including the widths, lengths, and lower and upper curved structures of the trileaflet template. Then, thin ePTFE membranes are trimmed into semilunar tricuspid shapes and connecting junctions to make handmade trileaflet valves. For customized applications, an intelligent assistant tool can provide optimal parameters to adjust the trileaflet sizes and conduit diameters for pulmonary valve reconstruction. Hence, surgeons can obtain the required trileaflet parameters for HTVC reconstructions. Then, using an experimental cardiopulmonary circulation loop system, the hemodynamic performance characteristics of customized conduits are validated under different hemodynamic statuses, including the regurgitation fraction $(R F)$, heart pump efficiency $(H P E)$, and pressure drop.

The most important task is to assess the hemodynamic status under different $H R$, blood flow volumes, and hypertension conditions. Using the completed parameter designs and by 
comparison with commercial valve stents, the design parameters are chosen to minimize the effects arising from variations in manufacturing on performance. Therefore, via statistical model validations, the quality of customized HTVCs is verified using the Taguchi method and likelihood degree estimation. ${ }^{(19-23)}$ Under normal conditions, less blood is regurgitated during the diastolic period as $R F \leq 20 \%$ (nominal-the-best, NTB). The cardiac output (CO) and pressure drop are related to the quantity of blood delivered to the lungs as $H P E \geq 80 \%$ (largerthe-better, LTB). Hence, the hemodynamic status of prosthetic trileaflet valves must be tested under normal and hypertension conditions in in vitro experiments, including the proposed customized HTVCs, Epic ${ }^{\mathrm{TM}}$ valves, mechanical heart valves, and a valveless condition. In performance tests, $R F$ and HPE are employed to determine whether the proposed HTVC has good performance characteristics compared with those of commercial valve stents. In addition, a sufficient pressure gradient (pressure drop) between the systolic and diastolic pressures across the valve can produce a forward blood flow to open the trileaflet valve under steady laminar flow (Reynolds number $<2000$ ). ${ }^{(24,25)}$ The pressure gradient is kept as large as possible (LTB). The dynamic motion (opening degree) can be observed using an endoscope inspection camera during the acceleration phase of the systolic stage.

Under the normal or hypertension condition, $R F$ should be as small as possible; $R F \approx 0 \%$ for no blood regurgitation and $R F \leq 20 \%$ for moderate regurgitation. In addition, $H P E \geq 80 \%$ indicates good outcomes for $\mathrm{CO}$ under both the normal and hypertension conditions. Hence, $H P E$ should also be as large as possible. In contrast to commercial products, the good quality attributes of HTVCs need to satisfy three specifications: (1) on target for $R F$ as minimum variation (NTB); (2) LTB for HPE and pressure drop; and (3) minimum variation for HPE in in vitro experiments in a cardiopulmonary circulation loop system with flow sensors, pressure transducers, and a data acquisition (DAQ) card. In this study, the Taguchi method is used to evaluate the valve efficiency via the $S / N$ ratio, including the nominal-the-best $\left(S / N_{N T B}\right)$, largerthe-better $\left(S / N_{L T B}\right)$, and smaller-the-better $\left(S / N_{S T B}\right)$ ratios. ${ }^{(19-23)}$ The $S / N$ ratios are used as measurable values instead of standard deviations to quantify the quality of manufactured conduits compared with the desired target through laboratory experiments. The Taguchi model is also a statistical method to improve the quality of the manufacturing processes and the designs for studying variations. A higher $S / N$ ratio indicates less variation (less quality loss) and more values with the desired customized specifications. The Taguchi method can identify the assigned optimal design parameters to obtain a better hemodynamic performance. To reduce the number of experimental tests, two ratios, $S / N_{N T B}$ and $S / N_{L T B}$, are used to analyze experimental data and identify the performance using likelihood degree estimation, ${ }^{(23)}$ then we compare the outcomes with those of Epic ${ }^{\mathrm{TM}}$ valve stents. The joint probability is specified as the likelihood degree for screening $S / N$ ratios under different $H R$, blood flow volumes, and hypertension conditions. Experimental results show that the proposed screening methods can be used to validate HTVC performance characteristics in clinical applications and verify the quality of customized specifications.

The remainder of this study is organized as follows: Section 2 describes the methodology, including the Taguchi and likelihood estimation methods. Sections 3 and 4 present the experimental system setup used, the results and discussion, a comparison with commercial valve stents, and the conclusion. 


\section{Materials and Methods}

\subsection{Taguchi method}

The Taguchi method is a statistical model to provide predictive knowledge on multivariable processes with a limited number of trials using different parameters. It uses the loss function to measure performance characteristics that deviate from the desired target values. Then, this loss function is transformed into $S / N$ ratios, including $S / N_{N T B}, S / N_{L T B}$, and $S / N_{S T B}$ ratios. These $S / N$ ratios can be used to validate the performance of valves with different sizes under different $H R$ and flow volumes. For a completed HTVC design, $R F, H P E$, and the pressure drop are generally used to evaluate pulmonary valve efficiency. $R F$ is estimated as the ratio between the forward stroke flow (systolic period) and the regurgitation flow (diastolic period) through the valve. ${ }^{(19)} H P E$ indicates the ability of the heart to pump an adequate amount of blood (out of the right ventricle) to the left and right lungs with each contraction, which is estimated from the forward stroke volume, pulmonary artery flow (PAF), and $H R{ }^{(19)}$ For each heartbeat, endsystolic and end-diastolic volumes, the indexes $R F$ and $H P E$, are computed under different $H R(60$ and 80 beats $/ \mathrm{min})$ and flow volumes $(40,50$, and $60 \mathrm{~mL}) \cdot{ }^{(9,16,19)} R F \leq 20 \%$ indicates moderate regurgitation, and $20 \%<R F<40 \%$ and $R F>40 \%$ indicate mild and severe regurgitations, respectively. $H P E \geq 80$ indicates good $\mathrm{CO}$ outcome. In fluid dynamics, the volume of flow per unit time (flow rate), $Q$, and the pressure drop $\Delta P(\mathrm{mmHg})$ can be estimated using the following equations: ${ }^{(23)}$

$$
\begin{gathered}
Q=V_{i n j} \times A \Rightarrow V_{i n j}=\frac{Q}{A} \approx \sqrt{\frac{P_{s y s}-P_{d i a}}{4}}=\sqrt{\frac{\Delta P}{4}}, \\
\Delta P=4\left(V_{i n j}\right)^{2},
\end{gathered}
$$

where $V_{i n j}$ and $A$ are the injection velocity and cross-sectional area $\left[A=\pi(D / 2)^{2} ; D(\mathrm{~mm})\right.$ is the inner diameter of the pulmonary valved conduit], and $P_{s y s}$ and $P_{d i a}$ are the systolic and diastolic pressures, respectively. The flow injection velocity is $25-140 \mathrm{~cm} / \mathrm{s}$ during the acceleration phase over the systolic stage $(<0.7 \mathrm{~s})$. Hence, the maximum pressure gradient $\Delta P$ imposed across the valve produces a forward flow, which can open the trileaflet valve. The peak wall-shear stress is estimated to be between 20 and $80 \mathrm{dyn} / \mathrm{cm}^{2}$. ${ }^{(24)}$ The HTVC yields a mean pressure with an average systolic pressure of $24.28 \mathrm{mmHg}$ and an average diastolic pressure of $16.55 \mathrm{mmHg}^{(19)}$ (normal values: systolic pressure, $20-30 \mathrm{mmHg}$; diastolic pressure, $8-12 \mathrm{mmHg}$; and mean pressure, $25 \mathrm{mmHg}$ ).

The Taguchi method is used with the aim of delivering a performance near the desired target, which overrides the specification limits. $R F$ can be used to deliver flow regurgitation near the target $(\leq 20 \%)$ with different diameters, $H R$, and flow volumes under a normal or hypertension condition; this case is referred to as NTB for minimum variation on the desired target. Optimal performance can be obtained when the variation is low and close to the desired target (variance reduction). To reduce the variability around a mean, $S / N R F, N T B$ is used as the NTB ratio to evaluate variations in $R F \%$ and is given as ${ }^{(19)}$ 


$$
S / N_{R F, N T B}=10 \times \log \left(\sum_{g=1}^{N}\left(\frac{R F \%_{\text {mean }}}{S_{R F}}\right)^{2}\right),
$$

where $R F \%_{\text {mean }}$ is the mean response value for $N$ experimental runs $g=1,2,3, \ldots, N(N=6$ in this study) and $S_{R F}$ is the standard deviation. The index $S / N_{R F, N T B}$ means that adjusting the mean on the target using the control design parameters of the handmade conduit has no effect on the desired target. $H P E \geq 80 \%$ indicates good valve performance. Given that small values are undesirable, $H P E$ should be as large as possible and must be higher than $80 \%$. $S / N_{H P E, L T B}$ is used as the LTB ratio to evaluate the HPE index to maximize the desired target and is given as

$$
S / N_{H P E, L T B}=-10 \times \log \left(\frac{1}{N} \sum_{g=1}^{N}\left(\frac{1}{H P E \%_{g}^{2}}\right)\right),
$$

where $H P E \% g$ is the $H P E$ index in the $g$ th running experiment, $g=1,2,3, \ldots, N$. $S / N_{H P E, N T B}$ is also used to evaluate variations in $H P E$ for the minimum variation of the desired target as

$$
S / N_{H P E, N T B}=10 \times \log \left(\sum_{g=1}^{N}\left(\frac{H P E \%_{\text {mean }}}{S_{H P E}}\right)^{2}\right),
$$

where $H P E \%_{\text {mean }}$ corresponds to the mean response for $N$ experimental runs and $S_{H P E}$ is the standard deviation. $\triangle P$ should also be as large as possible to produce a sufficient forward flow to open the trileaflet valve. $S / N_{P D, L T B}$ is used to evaluate a jet originating from the valvular orifice and is given as

$$
S / N_{P D, L T B}=-10 \times \log \left(\frac{1}{N} \sum_{g=1}^{N}\left(\frac{1}{\Delta P_{g}^{2}}\right)\right),
$$

where $\Delta P_{g}$ is the pressure gradient in the gth running experiment.

\subsection{Likelihood estimation method}

On the basis of the templates of the family of pulmonary leaflets shown in Fig. 1(a), two curved geometric lines, $C_{1}$ and $C_{2}$, and two vertical lines, $C_{3}$ and $C_{4}$, can be computed as shown in Refs. 16 and 19. In previous studies, ${ }^{(16,19)}$ intelligent methods, such as a metalearningbased intelligent model and a cascade correlation machine learning model, have been used to determine the optimal parameters $\left(L_{1}, L\right.$, and $\left.\mu\right)$ to design customized HTVCs for both child and adult patients. The performance of the HTVC is correlated with the optimal parameters $L_{1}$, $L$, and $\mu$ for our valve design. Obtaining the optimal parameters for making the valved conduit is necessary before the transcatheter pulmonary valve replacement (TPVR) treatment. Then, 
(a)

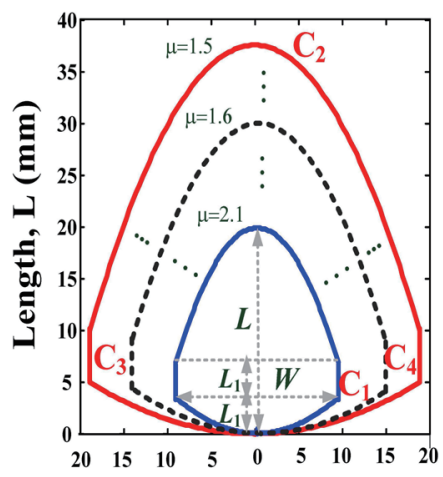

(c)

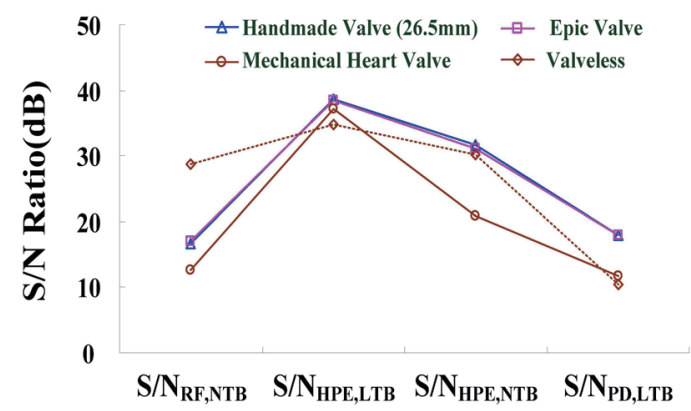

(b)

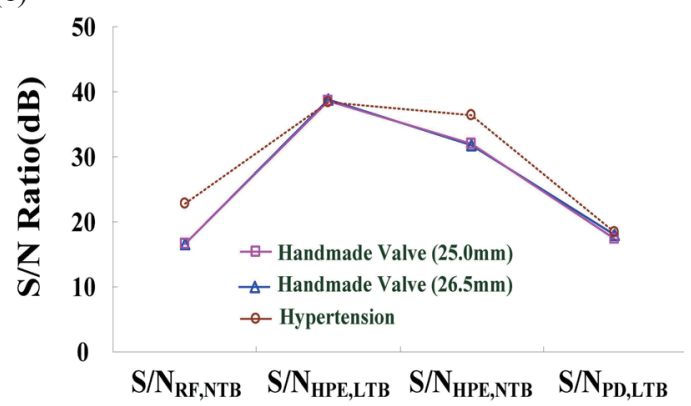

(d)

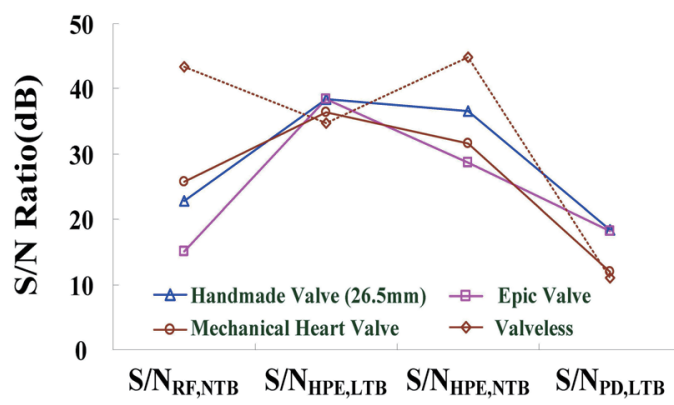

Fig. 1. (Color online) Templates of family of pulmonary leaflets and $S / N$ ratios for different valves. (a) Geometric shapes of family of pulmonary leaflets with different diameters, (b) $S / N$ ratios for handmade valved conduits with diameters of 25.0 and $26.5 \mathrm{~mm}$ under normal and hypertension conditions, (c) $S / N$ ratios for different valves under normal condition, and (d) $S / N$ ratios for different valves under hypertension condition.

the performance of the valved conduit should be analyzed to verify the hemodynamic status and valve efficacy. Hence, in contrast to the case of commercial valve stents, the performance characteristics of HTVCs with different diameters are validated under different $H R$, blood flow volumes, and normal and hypertension conditions using the Taguchi method. Through experimental biophysical tests, $S / N_{N T B}$ and $S / N_{L T B}$ indices for $R F, H P E$, and $\triangle P$ are used to measure the quality attributes that may deviate from desired target values, such as $R F \leq 20 \%$ for the NTB ratio, $H P E \geq 80 \%$ for the LTB and NTB ratios, and a sufficient $\triangle P$ for the LTB ratio, as shown in Figs. 1(b)-1(d). The ideal indices are expressed as

$$
\Phi=\left[\begin{array}{llll}
S / N_{R F, N T B}^{E p i c} & S / N_{H P E, L T B}^{E p i c} & S / N_{H P E, N T B}^{E p i c} & S / N_{P D, L T B}^{E p i c}
\end{array}\right]
$$

These four indices in Eq. (7), used as a gold standard, are estimated with the Epic ${ }^{\mathrm{TM}}$ valved stent under different $H R$, flow volumes, and normal conditions. Euclidean distances are used to screen the similarity between the HTVC and Epic ${ }^{\mathrm{TM}}$ commercial valve stents as follows:

$$
d_{1}=\sqrt{\left(S / N_{R F, N T B}-S / N_{R F, N T B}^{E p i c}\right)^{2}},
$$




$$
\begin{aligned}
& d_{2}=\sqrt{\left(S / N_{H P E, L T B}-S / N_{H P E, L T B}^{E p i c}\right)^{2}}, \\
& d_{3}=\sqrt{\left(S / N_{H P E, N T B}-S / N_{H P E, N T B}^{E p i c}\right)^{2}}, \\
& d_{4}=\sqrt{\left(S / N_{P D, L T B}-S / N_{P D, L T B}^{E p i c}\right)^{2}} .
\end{aligned}
$$

For the four indices, a joint probability accommodating the variance with $R F, H P E$, and $\triangle P$ is given as ${ }^{(23)}$

$$
\begin{gathered}
p=p_{1}\left(S / N_{R F, N T B}\right) p_{2}\left(S / N_{H P E, L T B}\right) p_{3}\left(S / N_{H P E, N T B}\right) p_{4}\left(S / N_{P D, L T B}\right) \\
=\exp \left(-\frac{1}{2}\left(\frac{d_{1}}{\sigma_{1}}\right)^{2}\right) \exp \left(-\frac{1}{2}\left(\frac{d_{2}}{\sigma_{2}}\right)^{2}\right) \exp \left(-\frac{1}{2}\left(\frac{d_{3}}{\sigma_{3}}\right)^{2}\right) \exp \left(-\frac{1}{2}\left(\frac{d_{4}}{\sigma_{4}}\right)^{2}\right) \\
\sigma_{j}=\frac{1}{4} \sum_{g=1}^{N} d_{j g}, j=1,2,3,4,
\end{gathered}
$$

where $\sigma_{j}$ is the standard deviation, which is estimated with the Epic ${ }^{\mathrm{TM}}$ valved stents under normal and hypertension conditions for $N$ experimental runs ( $\sigma=5$ in this study). A joint probability is also a statistical measurement that can calculate the likelihood of two or more events occurring simultaneously. When $p_{1}$ to $p_{4}$ are very similar, small Euclidean distances are obtained. The likelihood degrees with joint probabilities $p>0.66\left(p_{1}>0.90, p_{2}>0.90, p_{3}>0.90\right.$, and $\left.p_{4}>0.90\right)$ are used to screen the same performance of the four indices. Otherwise, differences obtained when the joint probability $p$ gradually decreases and approaches zero indicate a failed design or a defective valve. The Euclidean distances $d_{1}$ to $d_{4}$ are used to compare the differences between the four indices. Thus, we find that a marginal probability exceeding 0.66 separates the same performance from a different performance in performance validation. The probability $p$ deals with the likelihood of three phenomena occurring, $R F \leq 20 \%, H P E \geq 80 \%$, and a sufficient $\triangle P$. Therefore, in contrast to the case of Epic ${ }^{\mathrm{TM}}$ valved stents, this likelihood degree can offer a quantitative indication to evaluate the likelihood of HTVC efficiency under different hemodynamic statuses.

\section{Experimental Results}

\subsection{Setup of experimental system}

As presented in Fig. $2,^{(9-11,19)}$ an experimental biophysical system is established to simulate a pulmonary circulation loop system consisting of an artificial right ventricle, a primary pulmonary artery with the right and left pulmonary branches, pinch valve resistors, 


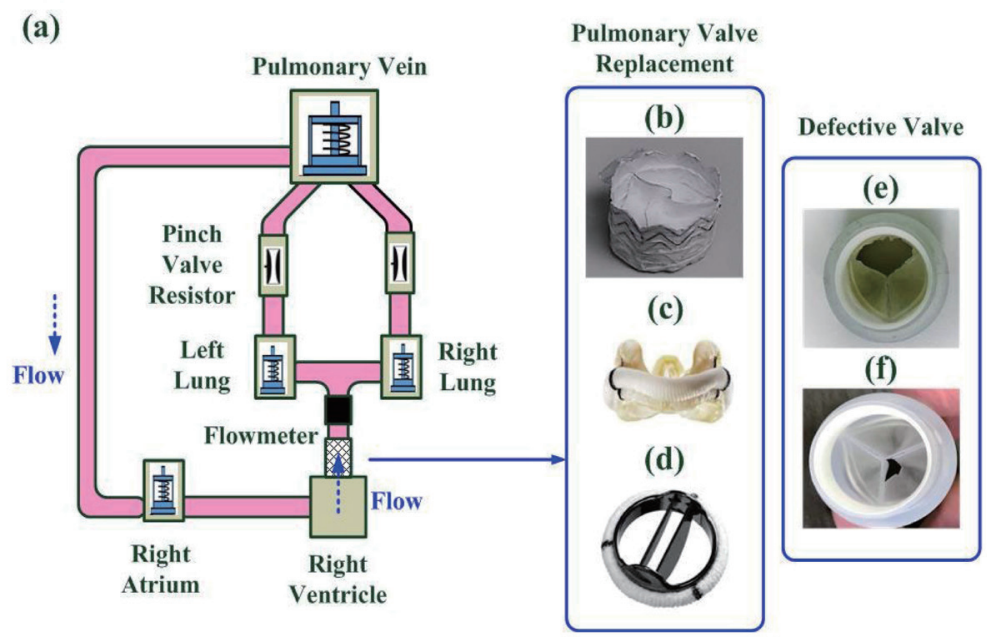

Fig. 2. (Color online) Setup of experimental system and artificial pulmonary valves. ${ }^{(9-11,19)}$ (a) Experimental

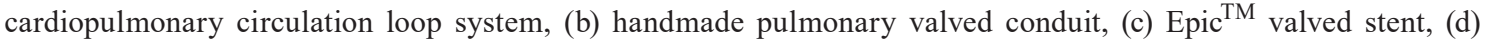
mechanical heart valve, (e) severely leaking conduit, and (f) miniature leaking valved conduit.

a pulmonary vein, and an artificial right atrium. ${ }^{(1,2)}$ The artificial right ventricle can produce blood pressure waves (HR of 60 and 80 beats/min) that propagate along the pulmonary arteries to the right and left lungs. The artificial pulmonary artery (APA) is made of $2.0-\mathrm{mm}$ thick silicone rubber with a T-shaped geometry, exhibits $0.2 \mathrm{~mL} / \mathrm{mmHg}$ volume compliance, and is connected to the artificial right and left lungs. The primary pulmonary artery allows the mimicking of blood flows of 40,50 , and $60 \mathrm{~mL}$ through the artificial pulmonary valve, as shown in Figs. 2(b)-2(f). Two sets of compliance chambers and pinch valve resistors are designed and attached to the distal ends of the right and left pulmonary arteries to mimic the resistance and compliance characteristics of the distal vasculature. The blood-mimicking fluid is made from water and glycerin (water:glycerin $=1.688: 1.000$; kinematic viscosity: $2.8-3.8 \mathrm{~m}^{2} / \mathrm{s}$; density: $\left.1.05-1.06 \mathrm{~kg} / \mathrm{m}^{3}\right)$ at $37 \pm 2{ }^{\circ} \mathrm{C}$.

The metering system used for further signal analysis consists of a transonic clamp-on flow sensor (ME16PXL, Transonic Systems; resolution, $10 \mathrm{~Hz} ; \pm 5 \mathrm{~mL} / \mathrm{min}$, bidirectional flow) and three pressure transducers (81A 006G Sensormate), which are used to acquire the ARV pressure, APA pressure, and APA flow via a DAQ card (National Instruments ${ }^{\mathrm{TM}}$, PCI-6259) connected to a laptop PC. Valvular insufficiency is evaluated on the basis of $R F \%, H P E \%$, and the pressure drop $\triangle P$ between systolic and diastolic pressures under different $H R$, flow volumes, and normal or hypertension conditions. For different $H R$ (60 and 80 beats $/ \mathrm{min})$ and flow volumes $(40,50$, and $60 \mathrm{~mL}$ ), an asymptomatic condition (right ventricle pressure $<60 \mathrm{mmHg}$ ) and a hypertension condition (right ventricle pressure $\geq 60 \mathrm{mmHg}$ ) are also considered to examine the hemodynamic conditions and valve performance with at least six experimental runs. The experimental biophysical model mimicking the human cardiopulmonary circulation system is set up in a laboratory located at the Heart Science and Medical Device Research Center at National Cheng Kung University, Hospital, National Cheng Kung University. 


\subsection{Validation of valve performance by Taguchi method}

As shown in Figs. 2(b)-2(d), the HTVC, Epic ${ }^{\mathrm{TM}}$ valve (St. Jude Medical), ${ }^{(10,11)}$ and mechanical heart valve (St. Jude Medical) ${ }^{(12,13)}$ were used to mimic pulmonary valve replacement in an APA. For the conduits with oversize diameters, $D=25.0 \mathrm{~mm}$ and $D=$ $26.5 \mathrm{~mm}$ were determined by CT pulmonary angiography, and then using intelligent methods, the related parameters of each leaflet were estimated: ${ }^{(1,2)} L_{1}=4.30 \mathrm{~mm}, L=26.83 \mathrm{~mm}$, and $\mu=1.75$ for the diameter of $25.0 \mathrm{~mm}$ and $L_{1}=4.58 \mathrm{~mm}, L=28.42 \mathrm{~mm}$, and $\mu=1.74$ for the diameter of $26.5 \mathrm{~mm}$. Each geometric shape of the family of pulmonary trileaflets is shown in Fig. 1(a). An HTVC was constructed using ePTFE for pulmonary valve reconstruction, as shown in Fig. 2(b). ${ }^{(9,19)}$ A flow sensor and pressure transducers were employed to obtain the pulmonary artery pressure (PAP) and PAF signals via a DAQ card connected to a laptop PC over at least 1 min, as can be seen in Fig. S1. For the different $H R s$ of 60 and 80 beats/min and the different flow volumes of 40,50 , and $60 \mathrm{~mL}$ under normal and hypertension conditions, the experimental results for $R F$, $H P E$, and pressure drop $(\mathrm{mmHg})$ are shown in Tables $1-3$, respectively. For example, as shown in Tables 1 and 2, for the diameter of $26.5 \mathrm{~mm}$, the average $R F$ is $15.01 \pm 2.22 \%(<20.00 \%)$ and the average $H P E$ is $86.10 \pm 2.22 \%(>80.00 \%)$ under the normal condition, and the average $R F$ is $17.20 \pm 1.25 \%$ and the average $H P E$ is $82.79 \pm 1.24 \%$ under the hypertension condition. The experimental results for both conditions indicate that the HTVC can decrease the regurgitation flow and increase the HPE. Using Eqs. (1) and (2), the forward flow rate and injection velocity can be used to estimate the pressure drop between the systolic and diastolic pressures. The pressure drop between the systolic and diastolic pressures under the normal condition is $\Delta P=7.89$ $\pm 3.02(\mathrm{mmHg})$ and that for the hypertension condition is $\Delta P=8.32 \pm 2.66(\mathrm{mmHg})$, as shown in Table 3.

By comparison, the proposed HTVCs with diameters of 25.0 and $26.5 \mathrm{~mm}$ showed numerical results similar to those of the Epic ${ }^{\mathrm{TM}}$ valved stents (the gold standard in this study) under

Table 1

Experimental results for $R F$ tests (six experimental runs) under normal and hypertension conditions with different $H R$ and flow volumes.

\begin{tabular}{|c|c|c|c|c|c|c|c|}
\hline \multirow[b]{2}{*}{ Valve type } & \multirow{2}{*}{$\begin{array}{l}\text { Experimental } \\
\text { run }\end{array}$} & \multirow{2}{*}{$\begin{array}{c}D \\
(\mathrm{~mm})\end{array}$} & \multirow[b]{2}{*}{ Hypertension } & \multirow{2}{*}{$\begin{array}{l}\text { Volume } \\
\text { (mL) }\end{array}$} & \multirow{2}{*}{$\begin{array}{c}H R \\
\text { (beat/min) }\end{array}$} & \multicolumn{2}{|c|}{$R F \%$} \\
\hline & & & & & & Mean & $\begin{array}{l}\text { Standard } \\
\text { deviation }\end{array}$ \\
\hline $\mathrm{I}$ & $1-6$ & 25.0 & $x$ & $40-60$ & $60-80$ & 14.58 & 2.15 \\
\hline II & $1-6$ & 25.0 & $x$ & $40-60$ & $60-80$ & 16.56 & 2.32 \\
\hline III & $1-6$ & 25.0 & $\times$ & $40-60$ & $60-80$ & 28.03 & 5.60 \\
\hline IV & $1-6$ & 25.0 & $\times$ & $40-60$ & $60-80$ & 44.08 & 1.45 \\
\hline I & $1-6$ & 26.5 & $\times$ & $40-60$ & $60-80$ & 15.01 & 2.22 \\
\hline II & $1-6$ & 26.5 & $\times$ & $40-60$ & $60-80$ & 16.57 & 2.32 \\
\hline III & $1-6$ & 26.5 & $\times$ & $40-60$ & $60-80$ & 28.03 & 6.52 \\
\hline IV & $1-6$ & 26.5 & $x$ & $40-60$ & $60-80$ & 45.59 & 1.66 \\
\hline I & $1-6$ & 26.5 & ० & $40-60$ & $60-80$ & 17.20 & 1.25 \\
\hline II & $1-6$ & 26.5 & ○ & $40-60$ & $60-80$ & 17.34 & 3.06 \\
\hline III & $1-6$ & 26.5 & o & $40-60$ & $60-80$ & 33.52 & 45.77 \\
\hline IV & $1-6$ & 26.5 & ○ & $40-60$ & $60-80$ & 1.75 & 0.31 \\
\hline
\end{tabular}

Note: I, Handmade Trileaflet Valve; II, Epic Valve; III, Mechanical Heart Valve; IV, Valveless 
Table 2

Experimental results for $H P E$ tests (six experimental runs) under normal and hypertension conditions with different $H R$ and flow volumes.

\begin{tabular}{|c|c|c|c|c|c|c|c|}
\hline \multirow[b]{2}{*}{ Valve type } & \multirow{2}{*}{$\begin{array}{l}\text { Experimental } \\
\text { run }\end{array}$} & \multirow[b]{2}{*}{$\begin{array}{c}D \\
(\mathrm{~mm})\end{array}$} & \multirow[b]{2}{*}{ Hypertension } & \multirow[b]{2}{*}{$\begin{array}{l}\text { Volume } \\
\text { (mL) }\end{array}$} & \multirow[b]{2}{*}{$\begin{array}{c}H R \\
\text { (beat/min) }\end{array}$} & \multicolumn{2}{|c|}{ HPE\% } \\
\hline & & & & & & Mean & $\begin{array}{l}\text { Standard } \\
\text { deviation }\end{array}$ \\
\hline I & $1-6$ & 25.0 & $x$ & $40-60$ & $60-80$ & 85.41 & 2.15 \\
\hline II & $1-6$ & 25.0 & $x$ & $40-60$ & $60-80$ & 83.42 & 2.32 \\
\hline III & $1-6$ & 25.0 & $x$ & $40-60$ & $60-80$ & 70.26 & 5.59 \\
\hline IV & $1-6$ & 25.0 & $x$ & $40-60$ & $60-80$ & 55.92 & 1.45 \\
\hline I & $1-6$ & 26.5 & $x$ & $40-60$ & $60-80$ & 86.10 & 2.22 \\
\hline II & $1-6$ & 26.5 & $x$ & $40-60$ & $60-80$ & 83.43 & 2.32 \\
\hline III & $1-6$ & 26.5 & $x$ & $40-60$ & $60-80$ & 71.97 & 6.52 \\
\hline IV & $1-6$ & 26.5 & $x$ & $40-60$ & $60-80$ & 54.40 & 1.66 \\
\hline I & $1-6$ & 26.5 & ○ & $40-60$ & $60-80$ & 82.79 & 1.24 \\
\hline II & $1-6$ & 26.5 & ○ & $40-60$ & $60-80$ & 82.65 & 3.06 \\
\hline III & $1-6$ & 26.5 & ○ & $40-60$ & $60-80$ & 66.47 & 1.75 \\
\hline IV & $1-6$ & 26.5 & $\circ$ & $40-60$ & $60-80$ & 54.23 & 0.31 \\
\hline
\end{tabular}

Note: I: Handmade Trileaflet Valve; II: Epic Valve; III: Mechanical Heart Valve; IV: Valveless

Table 3

Experimental results of pressure drop under normal and hypertension conditions with different $H R$ and flow volumes.

\begin{tabular}{|c|c|c|c|c|c|c|c|}
\hline \multirow[b]{2}{*}{ Valve type } & \multirow{2}{*}{$\begin{array}{l}\text { Experimental } \\
\text { run }\end{array}$} & \multirow[b]{2}{*}{$\begin{array}{c}D \\
(\mathrm{~mm})\end{array}$} & \multirow[b]{2}{*}{ Hypertension } & \multirow[b]{2}{*}{$\begin{array}{l}\text { Volume } \\
\text { (mL) }\end{array}$} & \multirow{2}{*}{$\begin{array}{c}H R \\
\text { (beat } / \mathrm{min} \text { ) }\end{array}$} & \multicolumn{2}{|c|}{$\Delta P(\mathrm{mmHg})$} \\
\hline & & & & & & Mean & $\begin{array}{l}\text { Standard } \\
\text { deviation }\end{array}$ \\
\hline I & $1-6$ & 25.0 & $x$ & $40-60$ & $60-80$ & 7.38 & 2.82 \\
\hline II & $1-6$ & 25.0 & $x$ & $40-60$ & $60-80$ & 8.15 & 2.73 \\
\hline III & $1-6$ & 25.0 & $x$ & $40-60$ & $60-80$ & 7.74 & 2.39 \\
\hline IV & $1-6$ & 25.0 & $x$ & $40-60$ & $60-80$ & 3.54 & 1.28 \\
\hline I & $1-6$ & 26.5 & $x$ & $40-60$ & $60-80$ & 7.89 & 3.02 \\
\hline II & $1-6$ & 26.5 & $x$ & $40-60$ & $60-80$ & 7.90 & 2.92 \\
\hline III & $1-6$ & 26.5 & $x$ & $40-60$ & $60-80$ & 3.89 & 1.49 \\
\hline IV & $1-6$ & 26.5 & $x$ & $40-60$ & $60-80$ & 3.36 & 1.25 \\
\hline I & $1-6$ & 26.5 & ○ & $40-60$ & $60-80$ & 8.32 & 2.66 \\
\hline II & $1-6$ & 26.5 & ○ & $40-60$ & $60-80$ & 8.15 & 2.73 \\
\hline III & $1-6$ & 26.5 & ○ & $40-60$ & $60-80$ & 3.96 & 1.31 \\
\hline IV & $1-6$ & 26.5 & ○ & $40-60$ & $60-80$ & 3.59 & 1.12 \\
\hline
\end{tabular}

Note: I: Handmade Trileaflet Valve; II: Epic Valve; III: Mechanical Heart Valve; IV: Valveless

different $H R$, flow volumes, and normal and hypertension conditions. A sufficient pressure gradient across the trileaflet valve can also produce a laminar flow for the forward flow. The HTVCs also show good outcomes that are better than those of the mechanical heart valve (average $R F>20 \%$, average $H P E<80 \%$ ). The performance was also validated by the Taguchi method as follows:

- To obtain the minimum variation from the desired targets $(R F=20 \%$ and $H P E=80 \%)$, the indexes $S /_{N R F, N T B}$ and $S / N_{H P E, N T B}$ are computed using Eqs. (3) and (5), respectively, with the mean values and standard deviations over six experimental runs.

- To maximize the objective function $(H P E \geq 80 \%$ ) and obtain a sufficient $\triangle P$, the indexes $S / N_{H P E, L T B}$ and $S / N_{P D, L T B}$ are computed using Eqs. (4) and (6), respectively, over six 
experimental runs under normal and hypertension conditions with different diameters, $H R$, and flow volumes.

Figures 1(b)-1(d) show the validation results for the proposed HTVC and commercial valve stents, including $S / N_{R F, N T B}, S / N_{H P E, L T B}, S / N_{H P E, N T B}$, and $S / N_{P D, L T B}$. To improve $R F$ and HPE, the desired targets are to reduce the deviations around means, $R F=20 \%$ and $H P E=80 \%$. For example, for the diameter of $26.5 \mathrm{~mm}, S / N_{R F, N T B}=16.60 \mathrm{~dB}$ and $S / N_{H P E N T B}=31.77 \mathrm{~dB}$ for the HTVC, which are similar to those of the Epic ${ }^{\mathrm{TM}}$ valved stent $(17.07$ and $31.77 \mathrm{~dB})$ used as the gold standard in this study, which indicates less variation from the desired target (less quality loss). The $S / N$ ratios are also better than those of the mechanical heart valve and the valveless condition under the normal condition, as shown in Fig. 1(c) and Table 4. For valve performance enhancement, $S / N_{H P E, L T B}=38.70 \mathrm{~dB}$ and $S / N_{P D, L T B}=17.95 \mathrm{~dB}$ of the HTVC are larger than those of the commercial valve stents and the valveless condition. As shown in Tables 4 and 5, for the HTVCs with diameters of 25.0 and $26.5 \mathrm{~mm}$, the performance characteristics are the same in terms of $R F, H P E$, and the minimum variation around the mean value under the normal condition, as shown in Fig. 1(b). However, slight deviations are observed: $S / N_{R F, N T B}=$ $22.77 \mathrm{~dB}$ and $S / N_{H P E, N T B}=36.49 \mathrm{~dB}$, indicating some disturbances around the mean under the hypertension condition. Hence, the HTVC can maintain a higher HPE and a sufficient pressure gradient, as shown in Tables 2 and 3, respectively.

In fluid dynamics analysis, a sufficient pressure gradient produces a forward flow across a trileaflet valve under a steady laminar flow with Reynolds numbers ranging from 300 to 450 (Reynolds number $<2000$ is a laminar flow and the mean Reynolds number of a pulmonary artery ranges from 275 to 1140$)^{(25)}$ and injection velocities ranging from 1.08 to $1.75 \mathrm{~m} / \mathrm{s}$ during the acceleration phase of the systolic stage, as shown in Fig. 3. Figure 4 shows the dynamic motion of a valve during the acceleration phase of the systolic stage obtained with an endoscope inspection camera at a rate of 30 frames/s. The proposed handmade trileaflet valve shows

Table 4

Numerical results of four $S / N$ ratios for proposed HTVC with diameter of $26.5 \mathrm{~mm}$ and commercial valve stents.

\begin{tabular}{|c|c|c|c|c|}
\hline \multirow[b]{2}{*}{$S / N$} & \multicolumn{4}{|c|}{ Valve type } \\
\hline & $\begin{array}{l}\text { Handmade } \\
\text { trileaflet valve }\end{array}$ & $\begin{array}{c}\text { Epic valve } \\
\text { (gold standard) }\end{array}$ & $\begin{array}{l}\text { Mechanical } \\
\text { heart valve }\end{array}$ & Valveless \\
\hline$S / N_{R F, N T B}(\mathrm{~dB})$ & 16.60 & 17.07 & 12.67 & 28.78 \\
\hline$S / N_{H P E, L T B}(\mathrm{~dB})$ & 38.70 & 38.42 & 37.14 & 34.71 \\
\hline$S / N H P E, N T B(\mathrm{~dB})$ & 31.77 & 31.77 & 20.86 & 30.31 \\
\hline$S / N_{P D, L T B}(\mathrm{~dB})$ & 17.94 & 17.95 & 11.80 & 10.53 \\
\hline
\end{tabular}

Table 5

Numerical results of four $S / N$ ratios for proposed HTVC with diameter of $25.0 \mathrm{~mm}$ and commercial valve stents.

\begin{tabular}{lcccc}
\hline & \multicolumn{4}{c}{ Valve type } \\
\cline { 2 - 5 }$S / N$ & $\begin{array}{c}\text { Handmade } \\
\text { trileaflet valve }\end{array}$ & $\begin{array}{c}\text { Epic valve } \\
\text { (gold standard) }\end{array}$ & $\begin{array}{c}\text { Mechanical } \\
\text { heart valve }\end{array}$ & Valveless \\
\hline$S / N_{R F, N T B}(\mathrm{~dB})$ & 16.63 & 17.07 & 13.99 & 29.66 \\
$S / N_{H P E, L T B}(\mathrm{~dB})$ & 38.63 & 38.43 & 36.93 & 34.95 \\
$S / N_{H P E, N T B}(\mathrm{~dB})$ & 31.98 & 31.11 & 21.98 & 31.72 \\
$S / N_{P D, L T B}(\mathrm{~dB})$ & 17.36 & 18.22 & 17.77 & 10.98 \\
\hline
\end{tabular}




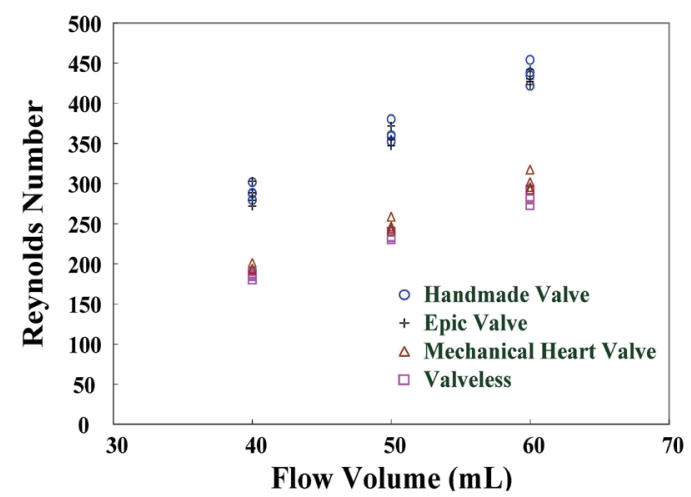

(a)

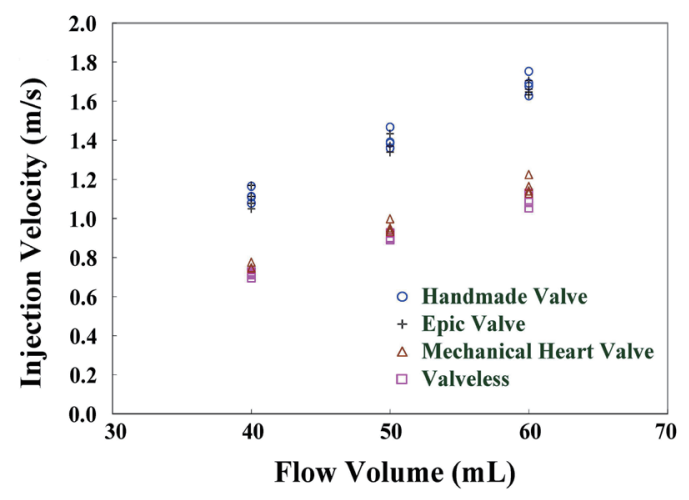

(b)

Fig. 3. (Color online) Fluid dynamics analysis. (a) Reynolds number versus flow volume (mL) and (b) injection velocity $(\mathrm{m} / \mathrm{s})$ versus flow volume $(\mathrm{mL})$. Note: Reynolds number $=\rho \times V_{i n j} \times D / \mu$, where $\rho$ is the fluid density $\left(\mathrm{kg} / \mathrm{m}^{3}\right), V_{i n j}$ is the injection velocity $(\mathrm{m} / \mathrm{s}), D$ is the inner diameter $(\mathrm{m})$, and $\mu$ is the fluid dynamic viscosity $\left(\mathrm{Ns} / \mathrm{m}^{2}\right)$ : $\mu=0.00743-0.01063 \mathrm{Ns} / \mathrm{m}^{2}$.

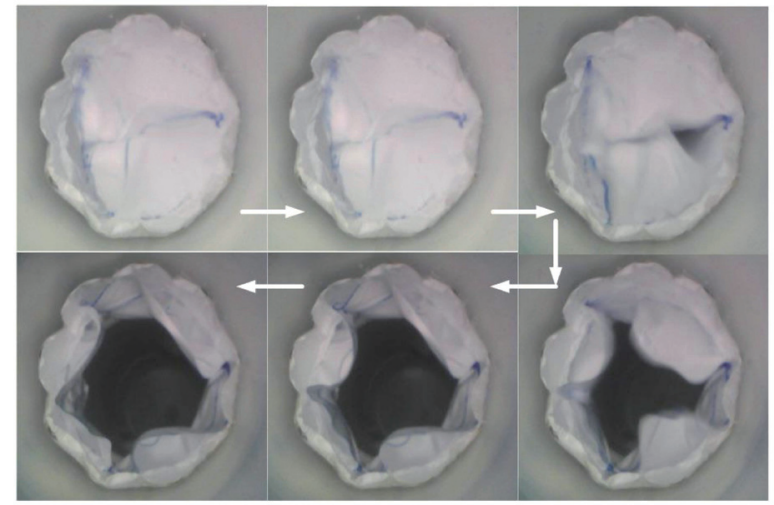

Fig. 4. (Color online) Dynamic motion of valve during acceleration phase of systolic stage.

a sufficient opening degree to allow a forward flow across it and closes tightly to prevent a regurgitation flow during each heartbeat. ${ }^{(26)}$

The Taguchi method can be easily applied by users with limited experimental runs ( $N=6$ for each set of experiments with different diameters, $H R$, and blood volumes) or knowledge of statistics to validate the effects of hemodynamic parameters on the performance characteristics, ${ }^{(27)}$ such as $R F, H P E$, and $\triangle P$. It used four $S / N$ ratios as quality characteristics in experimental data analysis, referring to the $\mathrm{Epic}^{\mathrm{TM}}$ valved stent as the gold standard. Under the normal condition (right ventricle pressure $<60 \mathrm{mmHg}$ ), eight sets of experiments were conducted, and the valve performance and hemodynamic status were also evaluated. The results were consistent between the handmade valves with different sizes and the Epic ${ }^{\mathrm{TM}}$ valve. Thus, examining the results for valves with different sizes can confirm the results of experiments to develop future applications for patients of different ages and extend the range of designs. 


\subsection{Validation of valve performance using likelihood estimation method}

As shown by the validation results in Fig. 5(a), likelihood degrees with a joint probability were employed to find the same performance characteristics for the handmade valves of different sizes (25.0 and $26.5 \mathrm{~mm}$ ), known as the close valve performance characteristics of $R F$, $H P E$, and $\triangle P$. The critical $S / N$ ratios of the Epic ${ }^{\mathrm{TM}}$ valved stent under the normal condition are $S / N_{R F, N T B}=17.07 \mathrm{~dB}, S / N_{H P E, L T B}=38.42 \mathrm{~dB}, S / N_{H P E, N T B}=31.11 \mathrm{~dB}$, and $S / N_{P D, L T B}=17.95 \mathrm{~dB}$, which are the gold standard in this study, as shown in Table 4. Comparing the handmade valves with the Epic ${ }^{\mathrm{TM}}$ valved stent, the higher likelihood degrees (0.94 and 0.91) of the former indicate that the handmade designs also showed the same performance characteristics despite the different diameters, $H R$, and blood volumes in each set of experimental runs. As shown in Fig. 1(d), referring to the outcome of the Epic ${ }^{\mathrm{TM}}$ valved stent under the normal condition, the $S / N$ ratios generally deviate under the hypertension condition (right ventricle pressure $>60 \mathrm{mmHg}$ ). The handmade valved conduit of $26.5 \mathrm{~mm}$ has variations in $S / N_{R F, N T B}(22.77 \mathrm{~dB})$ and $S / N_{H P E, N T B}(35.67 \mathrm{~dB})$, and the Epic ${ }^{\mathrm{TM}}$ valve also has larger variations in $S / N_{R F, N T B}(15.07 \mathrm{~dB})$ and $S / N_{H P E, N T B}(28.63 \mathrm{~dB})$. The mechanical heart valve shows insufficient ratios, namely, $S / N_{R F, N T B}(25.64 \mathrm{~dB}), S / N H_{P E, L T B}(36.45 \mathrm{~dB})$, and $S / N_{P D, L T B}(11.95 \mathrm{~dB})$, as can be seen in Table 6. As shown in Fig. 5(b), the likelihood degrees required to verify the promising performance

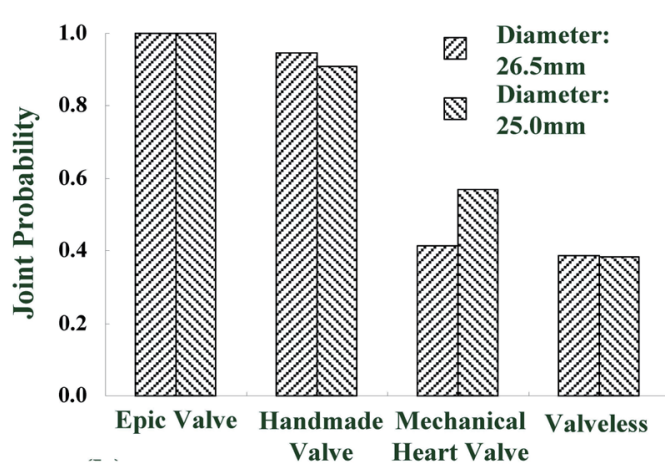

(a)

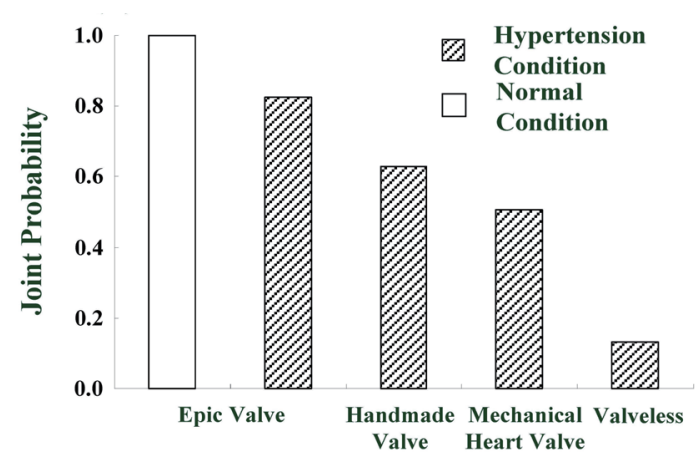

(b)

Fig. 5. Results of likelihood degree validation. (a) Likelihood degrees for handmade valves with different sizes and commercial valves under normal condition, and (b) likelihood degrees under normal and hypertension conditions.

Table 6

Numerical results of four $S / N$ ratios under hypertension condition for proposed handmade trileaflet valve conduit with diameter of $26.5 \mathrm{~mm}$ and commercial valve stents.

\begin{tabular}{lccccc}
\hline & \multicolumn{5}{c}{ Valve type } \\
\cline { 2 - 6 }$S / N$ & $\begin{array}{c}\text { Handmade } \\
\text { trileaflet valve }\end{array}$ & $\begin{array}{c}\text { Epic valve } \\
\text { (gold standard) }\end{array}$ & $\begin{array}{c}\text { Mechanical } \\
\text { heart valve }\end{array}$ & $\begin{array}{c}\text { Severe } \\
\text { leaking }\end{array}$ & Valveless \\
\hline$S / N_{R F, N T B}(\mathrm{~dB})$ & 22.77 & 15.07 & 25.64 & 23.52 & 43.38 \\
$S / N_{H P E, L T B}(\mathrm{~dB})$ & 38.36 & 38.34 & 36.45 & 35.58 & 34.68 \\
$S / N_{H P E, N T B}(\mathrm{~dB})$ & 35.67 & 28.63 & 31.59 & 27.08 & 44.86 \\
$S / N_{P D, L T B}(\mathrm{~dB})$ & 18.40 & 18.22 & 11.95 & 12.34 & 11.10 \\
\hline
\end{tabular}


characteristics under the hypertension condition are 0.82 and 0.65 . The likelihood degrees of similarity were computed using Eqs. (12) and (13). Although $R F(17.20 \% \pm 2.43 \%)$ and $H P E$ $(82.79 \% \pm 2.43 \%)$ are affected by symptomatic high pressures, the outcome also indicates good valve performance characteristics: $R F<20 \%, H P E>80 \%$, and mean $\triangle P=8.32 \pm 2.66 \mathrm{mmHg}$ (systolic/diastolic pressures: $51.20 / 41.71 \mathrm{mmHg}$ ). In contrast to those of the Epic ${ }^{\mathrm{TM}}$ valved stent and mechanical heart valve (likelihood degree $p_{M e c h}=0.50$ ), the performance characteristics of the handmade trileaflet valve $(26.5$ and $25.0 \mathrm{~mm})$ also show significant improvements in $R F$ and pumping efficacy under normal and hypertension conditions.

In addition, cases of valvular insufficiency, such as defective valves and the valveless condition, a miniature valve, and a valve with severe leaking, can decrease the systolic function in each cardiac cycle: $R F>20 \%$ and $H P E<80 \%$. As shown in Fig. 6(a), the patterns of the $S / N$ ratios show deviations in the four indexes. Hence, the likelihood degree with a joint probability has inverse relationships with the increases in poor quality levels from 0.54 to 0.38 , as seen in Fig. 6(b). For example, the likelihood degree for severe leaking (as shown in Table 6) can be computed as

$$
\begin{aligned}
& p_{\text {Seve }}=\exp \left(\frac{-1}{2}\left(\frac{23.52-17.07}{5}\right)^{2}\right) \exp \left(\frac{-1}{2}\left(\frac{35.58-38.42}{5}\right)^{2}\right) \\
& \exp \left(\frac{-1}{2}\left(\frac{27.08-31.77}{5}\right)^{2}\right) \exp \left(\frac{-1}{2}\left(\frac{12.34-17.95}{5}\right)^{2}\right) \approx 0.46
\end{aligned}
$$

The proposed likelihood degree model also offers a feasibility index for product quality screening and valve insufficiency assessment, such as in cases of valves with a poor design or defective valves.

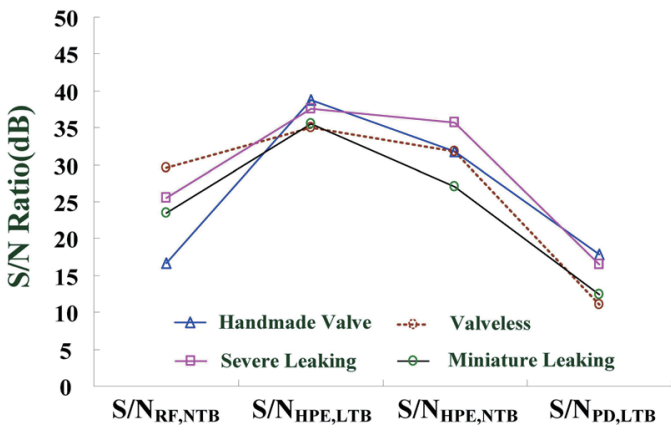

(a)

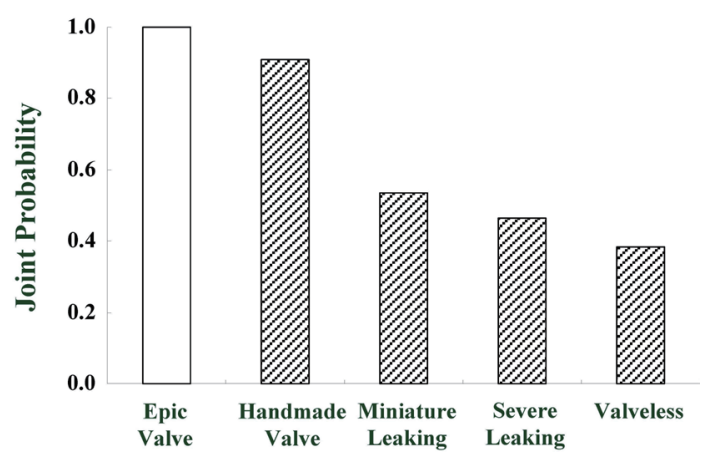

(b)

Fig. 6. (Color online) Likelihood degree validations for defective valves. (a) $S / N$ ratios for handmade valve, defective valves (miniature and severe leaking), and valveless condition. (b) Likelihood degrees for handmade valve, defective valves, and valveless condition. 


\section{Conclusion}

In this study, the performance characteristics of HTVCs were validated using the Taguchi and likelihood estimation methods for different diameters, $H R$, blood volumes, and normal and hypertension conditions. The hemodynamic performance characteristics of valves, including those of the proposed HTVCs and commercial products, were tested in an experimental cardiopulmonary circulation loop system. The Taguchi method was used to verify that the outcomes of the handmade valves satisfied the desired targets of $R F \leq 20 \%$ and $H P E \geq 80 \%$ with $S / N_{R F, N T B}$ and $S / N_{H P E, N T B}$ tests (minimum variation on the desired target), and the LTB ratio for $H P E$ and $\triangle P$ with $S / N_{H P E, L T B}$ and $S / N_{P D, L T B}$ tests (maximizing the desired target), respectively. The quality of the HTVCs under different hemodynamic conditions was similar to that of the Epic ${ }^{\mathrm{TM}}$ valved stent, which is considered as the gold standard. Some advantages of the Taguchi method are as follows: (1) it can be performed with experimental runs (six experimental runs in this study) fewer than that when using trial and error; (2) it can analyze the experimental data and identify the quality of the customized handmade valves; and (3) it can control the different diameters to validate the outcomes of customized handmade valves in terms of $R F, H P E$, and $\triangle P$. Experimental results also showed that the HTVCs can be used in clinical applications and for verifying the quality of manufactured valves using $S / N$ ratios and likelihood degrees $(>0.66)$. For future studies, in vivo animal experiments with HTVCs can be carried out to validate the mid-term and long-term follow-up effectiveness of the proposed strategy in order to prevent valve degeneration and repeated heart surgery.

\section{Acknowledgments}

This work was supported in part by the Ministry of Science and Technology, Taiwan, under contract numbers MOST 107-2221-E-006-156-MY2 (duration: August 1, 2018-July 31, 2020) and MOST 107-2221-E-167-029 (duration: August 1, 2018-October 31, 2019).

\section{References}

1 N. Holoshitz and Z. M. Hijazi: Interv. Cardiol. 5 (2013) 465. https://doi.org/10.2217/ICA.13.36 C2013Future Medicine Ltd.

2 W. A. Zoghbi, Maurice Enriquez-Sarano, E. Foster, P. A. Grayburn, C. D. Kraft, R. A. Levine, P. Nihoyannopoulos, C. M. Otto, M. A. Quinones, H. Pakowski, W. J. Stewatt, A. Waggoner, and N. J. Weissman: J. Am. Soc. Echocardiogr. 16 (2003) 777. https://doi.org/10.1016/S0894-7317(03)00335-3

3 Y. Ko, K. Morita, T. Abe, M. Nakao, and K. Hashimoto: World J. Pediatric Congenital Heart Surg. 6 (2015) 502 https://doi.org/10.1177/2150135115598209

4 Y. Y. Kim and E. Ruckdeschel: Heart 102 (2016) 1520. https://doi.org/10.1136/heartjnl.2015-309067

5 M. Nakagawa, S. Katou, M. Ichinose, S. Nobe, H. Yonemochi, I. Miyakawa, and T. Saikawa: J. Electrocardiol. 37 (2004) 47. https://doi.org/10.1016/j.jelectrocard.2003.10.007

6 P. Nathani, S. Shetty, and Y. Lokhandwala: J. Assoc. Physicians India 2007 (Suppl. 2007) 33. https://www. ncbi.nlm.nih.gov/pubmed/18368865

7 T. W. Lin, J. N. Wang, C. D. Kan, and Y. J. Yang: J. Thorac. Cardiovasc. Surg. 148 (2014) 1753. https://doi. org/10.1016/j.jtcvs.2014.05.083

8 M. T. Lin, J. K. Wang, P. Y. Wu, C. W. Lu, T. I Chang, and Y. S. Chen: Soc. Thorac. Surg. 12 (2016) E541. https://doi.org/10.1016/j.athoracsur.2016.04.093

9 C. D. Kan, J. N. Wang, W. L. Chen, P. J. Lu, M. Y. Chan, C. H. Lin, and W. C. Hsieh: J. Thorac. Cardiovasc. Surg. 155 (2018) 765. https://doi.org/10.1016/j.jtcvs.2017.09.049 
10 EpicTM Stented Tissue Valve with LinxTM AC Technology, http://www.cardion.cz/file/9/epic-stented-tissuevalve-with-linx-ac-technology.pdf (accessed 2013).

11 P. S. Myken and O. Bech-Hansen: J. Thorac. Cardiovasc. Surg. 137 (2009) 76. https://doi.org/10.1016/ j.jtcvs.2008.05.068

12 T. Shipkowitz, J. Ambrus, J. Kurk, and K. Wickramasinghe: J. Heart Valve Dis. 11 (2002) 275. https://www. ncbi.nlm.nih.gov/pubmed/12000172

13 Z. Feng, T. Nakamura, T. Fujimoto, and M. Umezu: Arti. Organs 26 (2002) 32. https://doi.org/10.1046/j.15251594.2002.06833.X

14 W. B. Eichinger, I. M. Hettich, D. J. Ruzicka, K. Holper, C. Schricker, S. Bieiziffer, and R. Lange: Ann. Thorac. Surg. 86 (2008) 1204. https://doi.org/10.1016/j.athoracsur.2008.05.058

15 P. Loyalka, M. Schechter, A. Nascimbene, A. S. Raman, C. A. Ilieascu, I. D. Gregoric, and B. Kar: Texas Heart Inst. J. 43 (2016) 341. https://doi.org/10.14503/THIJ-15-5310

16 C. D. Kan, W. L. Chen, C. H. Lin, J. N. Wang, P. J. Lu, M. Y. Chan, and J. T. Wu: IEEE Access 6 (2018) 2138. https://doi.org/10.1109/ACCESS.2018.2815599

17 E. Yamashita, M. Yamagishi, T. Miyazaki, Y. Maeda, Y. Yamamoto, N. Kato, H. Hongu, and H. Yaku: Ann. Thorac. Surg. 102 (2016) 1336. https://doi.org/10.1016/j.athoracsur.2016.03.027

18 C. H. Chiang, M. L. Yeh, W. L. Chen, and C. D. Kan: Ann. Vasc. Surg. 31 (2016) 196. https://doi.org/10.1016/ j.avsg.2015.10.007

19 C. D. Kan, W. L. Chen, C. H. Lin, J. N. Wang, P. J. Lu, M. Y. Chan, and J. T. Wu: IEEE Access 6 (2018) 7088. https://doi.org/10.1109/ACCESS.2017.2782686

20 B. G. Irad: IEEE Trans. Reliab. 54 (2005) 381. https://doi.org/10.1109/TR.2005.853280

21 B. G. Irad, R. Katz, and Y. Bukchin: IIE Trans. 40 (2008) 907. https://doi.org/10.1080/07408170701775094

22 D. Fratilia and C. Caizar: J. Cleaner Prod. 19 (2011) 640. https://doi.org/10.1016/j.jclepro.2010.12.007

23 C. D. Kan, W. L. Chen, C. H. Lin, Y. C. Mai, and M. J. Wu: Technol. Healthcare Care. 25 (2017) 887. https:// doi.org/10.3233/THC-160474

24 S. H. Gharaie and Y. Morsi: Int. J. Artif. Organs 38 (2015) 259. https://doi.org/10.5301/ijao.5000413

25 G. W. Henry, T. A. Johnson, J. I. Ferreiro, H. S. Hsiao, C. L. Lucas, B. A. Keagy, M. E. Lores, and B. R. Wilcox: Cardiovasc. Res. 18 (1984) 620. https://doi.org/10.1093/cvr/18.10.620

26 W. L. Chen, T. L. Yang, and C. D. Kan: J. Thorac. Cardiovasc. Surg. 156 (2018) 1639. https://doi.org/10.1016/ j.jtcvs.2018.05.034

27 S. K. Karna and R. Sahai: Int. J. Eng. Math. Sci. 1 (2012) 11. https://www.ijser.org/researchpaper/AnOverview-of-Taguchi-Method-Evolution-Concept-and-Interdisciplinary-Applications.pdf 


\section{Appendix}

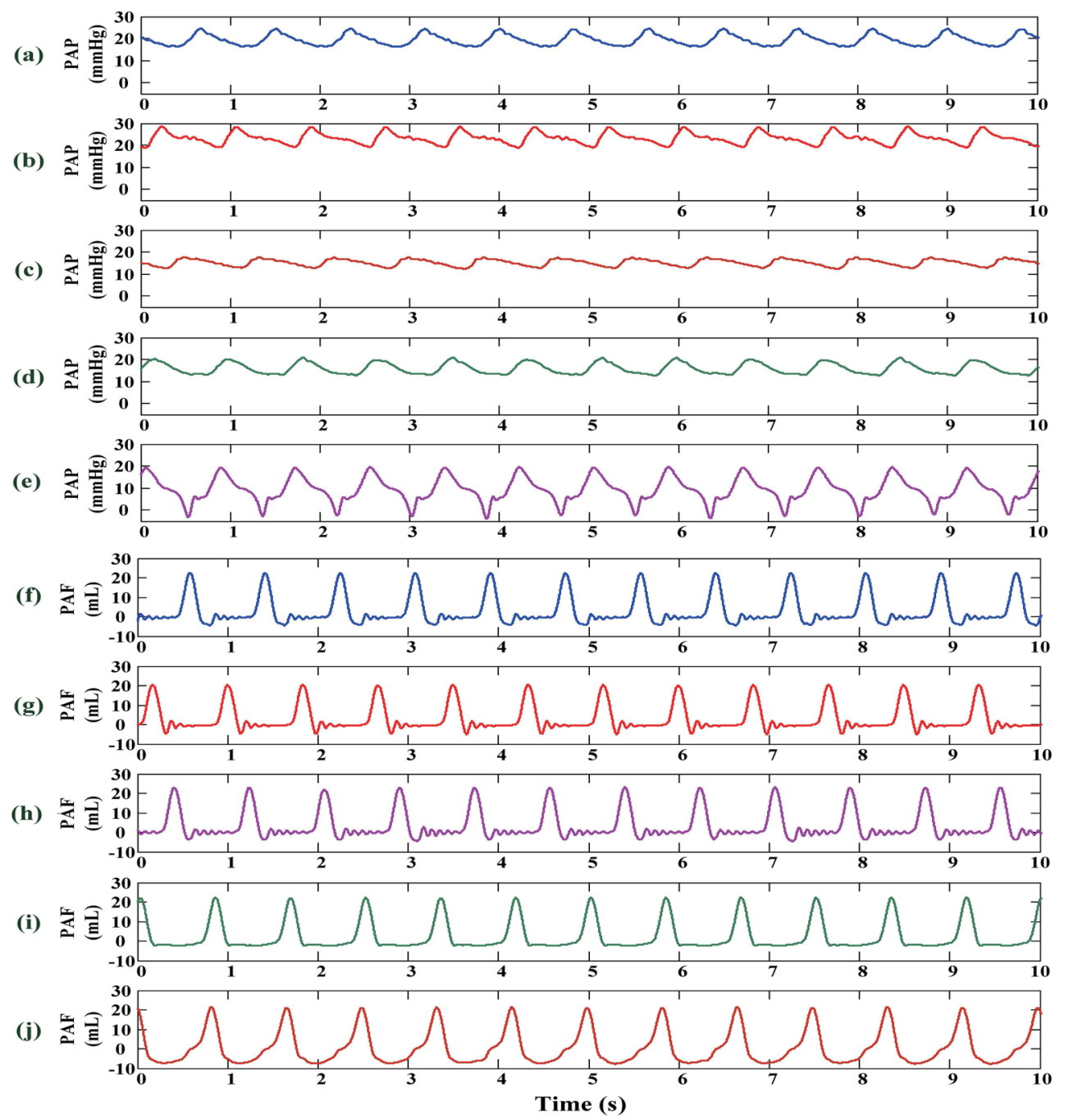

Fig. S1. (Color online) Raw data of PAP and PAF with 60 beats/min and $60 \mathrm{~mL}$ flow volume under normal condition. (a) and (f) PAP and PAF for handmade trileaflet valve, (b) and (g) PAP and PAF for Epic ${ }^{\text {TM }}$ valved stent, (c) and (h) PAP and PAF for mechanical heart valve, (d) and (i) PAP and PAF for defective valve (miniature leaking), and (e) and (j) PAP and PAF for valveless condition, respectively. 